\title{
The distribution of mucosal antibodies in the bowel of patients with Crohn's disease
}

\author{
F. H. Y. GREEN AND H. FOX
}

From the Department of Pathology, University of Manchester

SUMMARY Specimens of intestine from 24 patients with histologically proven Crohn's disease have been studied by an immunofluorescent technique for the distribution of immunoglobulins A, G, M, E, and D. A marked reduction of IgA in lymphoid and epithelial cells was noted in those areas of the bowel which, although histologically affected, were not ulcerated; in the histologically unaffected areas of the bowel the distribution of $\operatorname{IgA}$ was similar to that found in control sections of normal bowel. There was an increase in IgM in the affected areas but the numbers of lymphoid cells staining for IgG, IgE, and IgD did not differ from those found in normal controls; in 12 cases of Crohn's disease, however, IgG appeared to be present in bound form on the surface of, and in, the epithelial cells.

It is postulated that the local deficiency in $\operatorname{IgA}$ in the affected areas may be secondary to a local cell-mediated immune reaction, to a focal metaplasia of the surface epithelium, or to patchy bindings of anticolon antibodies to the surface epithelium. The effect of this local deficiency of $\operatorname{IgA}$ will be to allow for an increased focal entry of antigenic material into the bowel wall, and it is suggested that many of the pathological features of Crohn's disease are explicable on the basis of deep penetration of a variety of antigens.

The existence of a distinct local immune mechanism for mucosal surfaces based on a secretory form of IgA has been well documented (Tomasi, 1970; Tomasi and DeCoteau, 1970). This secretory immunoglobulin system plays a particularly vital role in the immune response of the gut to the foreign antigens to which it is being constantly exposed and it is therefore not surprising that attention is being increasingly paid to alterations in the population of bowel immunocytes in a wide range of gastrointestinal diseases. A limited amount of information is available about changes in the numbers of immunoglobulin-containing cells in the bowel in patients with Crohn's disease (Søltoft, 1969; Schofield, Deodhard, and Turnbull, 1969) but there does not appear to have been any systematic study of the distribution of mucosal antibodies in this condition.

In this paper we report a study of the immunoglobulin-containing cells of the bowel in patients with Crohn's disease, particular attention being paid

Received for publication 13 November 1974. to the relative numbers of immunocytes in the affected areas of the gut.

\section{Materials and Methods}

\section{SPECIMENS}

Surgically resected unfixed specimens of both large and small intestine were obtained from 24 patients with Crohn's disease. These represented, as far as possible, a consecutive series and no attempt was made to select cases; as, however, the material was all obtained from patients requiring surgical intervention the group was only representative of the relatively more severe form of the disease. The mean age of the patients was 39 years and symptoms had been present for an average of seven years. Most had received steroid therapy at some time and five patients had been given a course of azathioprine, though none were receiving such treatment at the time of operation. Half the patients had predominantly large bowel disease whilst in the other half the small bowel was predominantly involved.

Up to 10 blocks were taken from the fresh specimens, the number depending upon the size of 
the specimen and the anatomical extent of the lesions. In all cases an attempt was made to take blocks from the resection lines, the ulcerated areas, the affected areas without mucosal ulceration (as assessed by thickening of the wall), and the unaffected areas. The accuracy of the macroscopic assessment was checked by subsequent histological study. In addition, blocks were taken, when possible, from attached mesenteric lymph nodes. Control blocks of normal intestine were obtained from both large and small bowel; most of the normal large bowel controls were taken from colons resected for carcinoma, the blocks being selected from areas well away from the tumour, whilst the normal small intestinal controls were made up partly of blocks from portions of terminal ileum attached to colons resected for tumour and partly of peroral jejunal biopsies in which no pathological abnormality could be found.

The blocks were snap frozen in isopentane cooled by solid carbon dioxide and serial sections were then cut in an open top cryostat at a thickness of $5 \mu$. Sections were fixed in acetone for three minutes and then either stained immediately or stored at $-20^{\circ} \mathrm{C}$ for up to 2 weeks.

\section{ANTISERA}

Specific freeze-dried antisera prepared in sheep against human IgA, IgG, and IgM and conjugated with fluorescein and isothiocyanate were obtained from Burroughs Wellcome and fluorescein-conjugated antihuman IgE, from Miles Yeda Ltd. In addition, unconjugated antihuman IgA, IgG, IgE, and IgM for use in blocking controls were obtained from the same sources. Unconjugated swine antihuman IgD and sheep antihuman secretory piece were obtained from Nordic Pharmaceuticals and Diagnostics, and fluorescein-conjugated rabbit antiswine immunoglobulin and rabbit antisheep immunoglobulin were obtained from the same source. All the antisera had been rendered monospecific at source by absorption techniques and their specificity confirmed by the commercial firms supplying them both before and after conjugation by immunoelectrophoresis. The secretory piece antisera showed no reaction against pooled human serum on immunodiffusion. Before use the antisera were reconstituted with distilled water and diluted 1 in 10 with isotonic saline.

\section{Staining}

\section{DIRECT TECHNIQUE}

The acetone-fixed sections were washed in two successive baths of buffered saline containing $0.02 \mathrm{M}$ Sorensen buffer at $\mathrm{pH} \mathbf{7 \cdot 2}$. The sections were dried and covered with two drops of conjugated antisera; these were then incubated in a moist chamber at room temperature for $\mathbf{3 0}$ minutes after which excess dye was removed by gentle dipping in successive baths of phosphate-buffered saline for 15 minutes. The slides were mounted in buffered glycerine at pH 7.2.

INDIRECT TECHNIQUE

After washing in buffered saline as described above the slides were incubated for $\mathbf{3 0}$ minutes in a moist chamber with two drops of the unconjugated antisera to IgD or secretory piece. The sections were then washed in buffered saline for 15 minutes, carefully blotted dry and covered with conjugated antisera to swine for IgD and to sheep for secretory piece. After further incubation and washing they were mounted in buffered glycerine.

In addition to the above staining techniques serial sections were cut and stained with haematoxylin and eosin.

\section{CONTROLS}

Unstained sections mounted in buffered glycerine were used for the estimation of autofluorescence. Specificity of the staining of each conjugate in the direct technique was confirmed by a blocking technique in which parallel sections were incubated, as above, with unconjugated antihuman IgA, IgG, IgM, or IgE; after washing in buffered saline the appropriate conjugated antiglobulin was added for a further 20 minutes. In the indirect technique a parallel section was incubated with the conjugated antisera only, to control for non-specific fluorescence.

\section{MICROSCOPY}

For fluorescence microscopy a Leitz Ortholux microscope fitted with a Phillips CS ultraviolet lamp, a GB exciter filter, and a K5 30 suppression filter was used. The microscope was fitted with a Leitz Orthomat camera and photographs were taken using Pan Plus X film.

The sections were initially examined under low power $(\times 40)$ to give an overall impression of the distribution of fluorescent cells. At a higher power ( $\times 90$ ), and using an eye-piece grid to exclude areas other than the lamina propria, the number of specifically fluorescent cells was counted in a known area; an average of at least eight high-power fields were examined in each section. Epithelial fluorescence was roughly quantitated using a 0 to +++ scale. The identity of the specifically fluorescent cells was checked by photographing selected areas and then restaining the section with haematoxylin and eosin. 
QUANTITATION OF RESULTS

The cell counts were expressed as numbers per unit area of lamina propria which at the magnification employed was $0.0748 \mathrm{sq} \mathrm{mm}$.

\section{Results}

\section{LIGHT MICROSCOPY}

The diagnosis of Crohn's disease was made according to the pathological guidelines laid down by LockhartMummery and Morson (1964), Williams (1964), and Schofield and Fox (1967). Cases not fulfilling these rigid criteria were excluded. In all the acceptable cases studied there was evidence of active disease and epithelioid granulomata were seen in 18 of the 24 cases. The inflammatory infiltrate usually consisted of a mixture of lymphocytes and plasma cells, with the former predominating.

\section{FLUORESCENT MICROSCOPY}

Comparison of the results obtained for large and small intestine, both for the cases of Crohn's disease and for the control cases, showed no significant difference between these two sites for any of the parameters studied. For clarity of presentation therefore the results obtained from these two sites have been combined.

DISTRIBUTION OF IMMUNOGLOBULIN-CONTAINING CELLS AND SECRETORY PIECE

In the bowel from the control cases there was the usual predominance of IgA-containing cells in the plasma cell population of the lamina propria and of IgA in the mucosal epithelial cells. The epithelial IgA was seen along the basement membrane, in the intercellular spaces, and in the apical part of the cytoplasm of the epithelial cells; it tended to be maximal in the crypts and in the glands. No epithelial fluorescence was seen in the sections from control cases when stained for IgG, IgD, or IgE though occasional slight fluorescence was seen when stained for IgM. The specifically fluorescing cells of the lamina propria tended to be most numerous around the crypts and in the deeper parts of the lamina propria with lesser numbers adjacent to the surface epithelium. The ratio of IgA-IgM-IgG-containing cells was $6: 2: 1$. The number of cells containing IgD or IgE was too small to quantitate. Staining for secretory piece gave a positive result throughout the mucosa of the small and large bowel; secretory piece was localized to the surface mucous coat, the apical cytoplasm of the enterocytes and goblet cells, and to the intercellular spaces, and was not demonstrable along the epithelial basement membrane or in the lamina propria.

In cases of Crohn's disease the histologically unaffected areas showed a slight increase in the number of IgA-containing cells in the lamina propria whilst the distribution of IgA in the epithelium was similar to that seen in sections from the control cases (fig 1). A definite increase in the number of IgM-containing cells was observed in these areas and their numbers were approximately equal to those of the IgA-containing cells; in addition, there was marked epithelial fluorescence when stained for IgM (fig 2) in 19 of the 24 cases, this having a similar distribution to that of the epithelial IgA. The distribution of IgG-, IgD-, and IgE-containing cells in the unaffected areas was similar to that seen in control cases. The counts of immunoglobulincontaining cells per unit area of the lamina propria in the unaffected areas are shown in figure 3. The distribution of secretory piece in these areas was similar to that seen in control cases (fig 4) and was present in all cases.

Sections from the areas showing histological evidence of Crohn's disease but with an intact mucosa showed a marked decrease in the number of IgA-containing cells in the lamina propria (fig 5), with, in some cases, several fields having to be examined before a specifically fluorescent cell of this type could be found. Although there was considerable variation between one case and another in the number of IgA-containing cells there was little variation between different blocks from the same case. Intra-epithelial IgA was decreased proportionally to the reduced number of IgA-containing cells and in many areas was completely absent. The

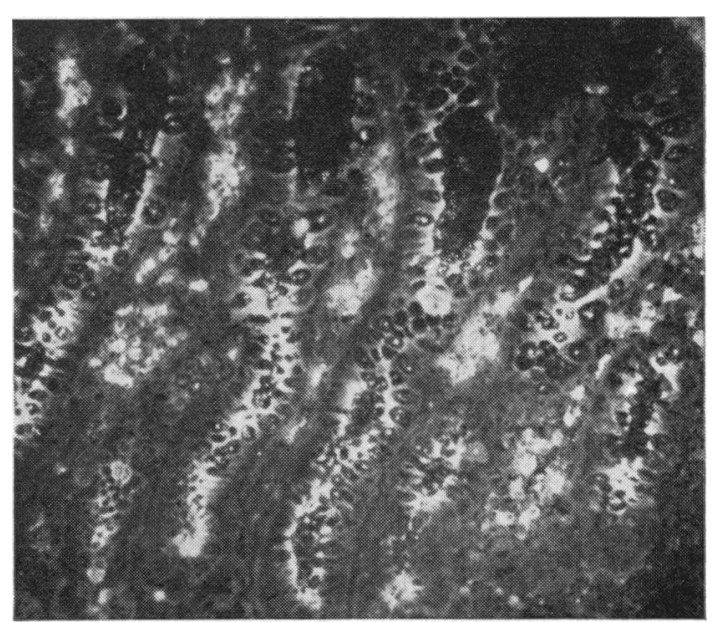

Fig 1 Crohn's disease. Histologically unaffected area of the bowel after incubation with anti-IgA. There is a slightly increased number of IgA-containing cells in the lamina propria and a normal distribution of $\operatorname{Ig} A$ in the epithelium $(\times 90)$ 


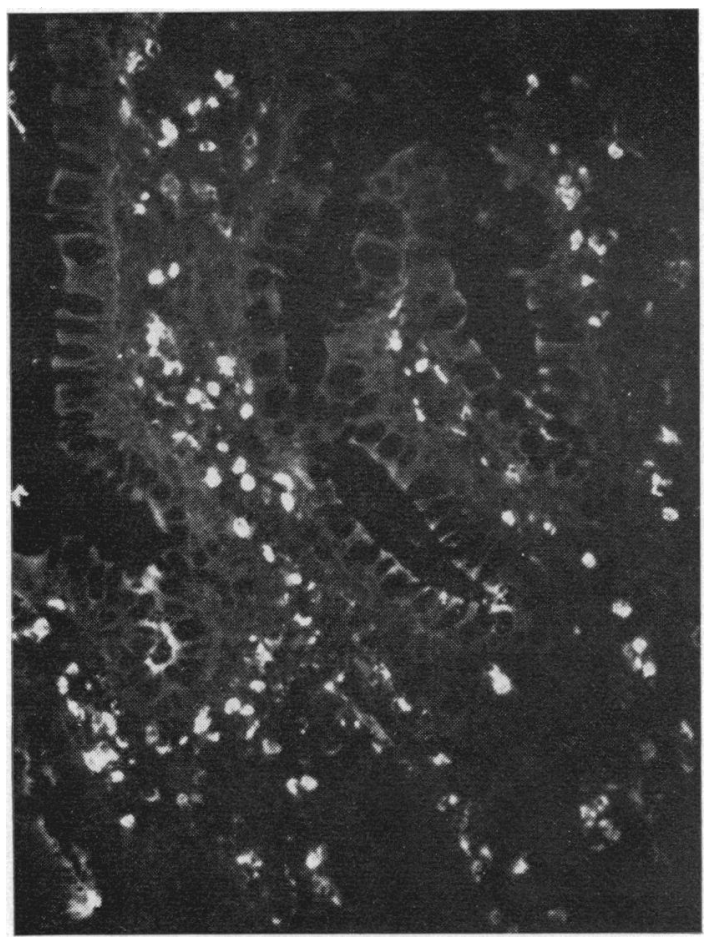

Fig 2 Crohn's disease. Histologically unaffected area of the bowel after incubation with anti-IgM. There is a moderate number of IgM-containing cells in the lamina propria and marked epithelial fluorescence $(\times 90)$.

number of IgM-containing cells in the affected areas was also decreased relative to the numbers seen in the unaffected areas but not relative to the numbers found in control cases; epithelial IgM was largely absent from the affected areas whilst the numbers of IgG-, IgD-, and IgE-containing cells did not differ significantly from those in control cases or in the unaffected areas of Crohn's cases. The counts of the various immunocytes per unit area of the lamina propria of the affected areas are shown in figure 6.

The epithelium of the affected areas stained positively for secretory piece (fig 7) which tended to be distributed uniformly throughout the cells and not concentrated in the apical cytoplasm as was the case in control cases and in the unaffected areas from cases of Crohn's disease. In addition, in the affected areas, marked linear staining of the luminal surface and apical cytoplasm of the enterocytes was seen with anti-IgG in 12 of the 24 cases (fig 8). In the unaffected areas of the same cases the apical part of the basal cells of the crypts and glands also fluoresced with anti-IgG. The intensity of this fluorescence was

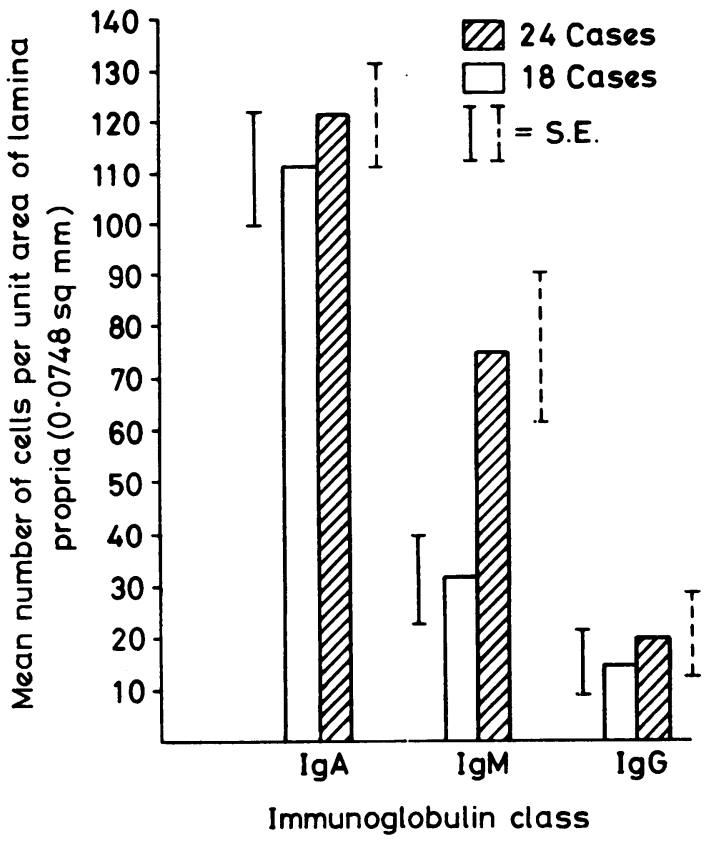

Fig 3 Mean number of immunoglobulin-containing cells in the histologically unaffected areas of the bowel of patients with Crohn's disease compared with normal controls.

only slightly diminished by prewashing the unfixed sections in buffered saline for 10 minutes.

In the ulcerated areas of the bowel in the cases of Crohn's disease the inflammatory infiltrate in the submucosa and muscle coat contained a moderate number of IgA-, IgM-, and IgG-containing cells, in that order. Lymphoid follicles staining for one immunoglobulin class were commonly seen in the submucosa, subserosa, and in the lymph nodes draining the affected areas; these were predominantly formed of IgA-containing cells.

\section{Discussion}

In the majority of cases of Crohn's disease there appears to be a focal reduction in detectable IgA, both in the lymphoid cells of the lamina propria and in the epithelial cells; this abnormality is confined to the areas showing histological abnormalities and is most marked in those in which the mucosa is either intact or only partially ulcerated. This abnormality of the local secretory immunoglobulin system does not appear to have been previously noted in Crohn's disease and is unlikely to be simply a dilution effect due to local oedema, for the total number of lymphoid cells in the affected area was, as assessed in haematoxylin- and eosin-stained sec- 


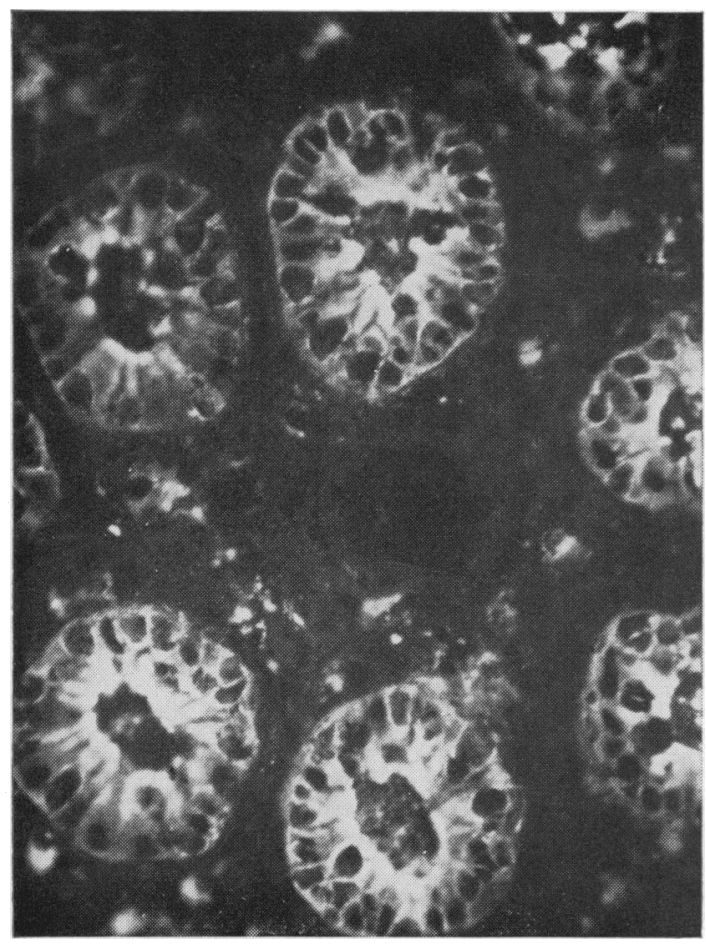

Fig 4

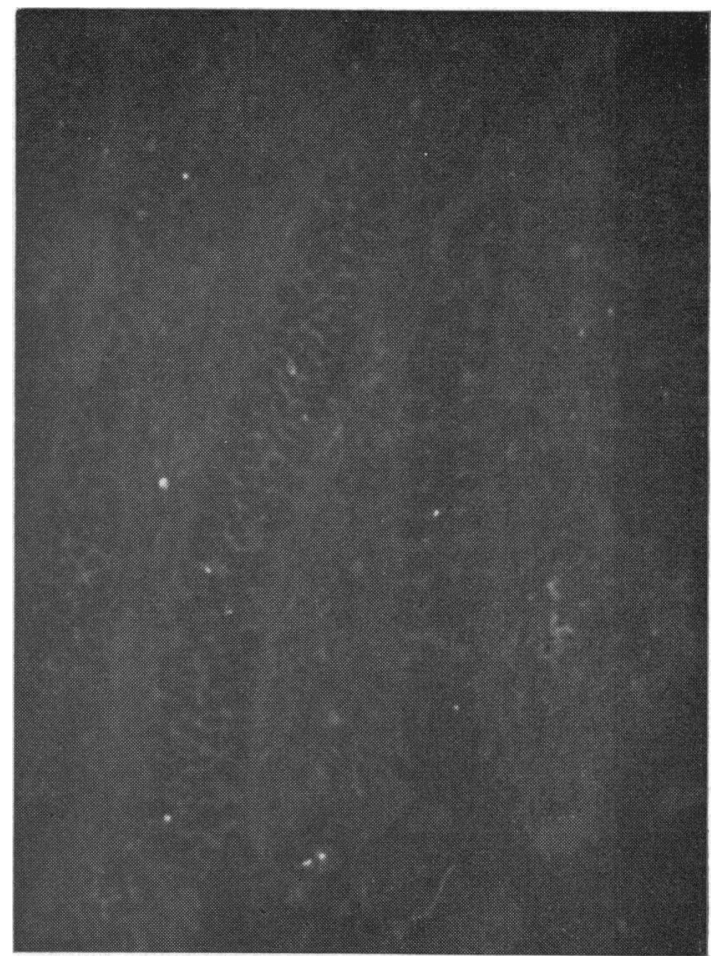

Fig 5

Fig 4 Immunofluorescent localization of secretory piece in the epithelial cells of unaffected areas of the bowel of a patient with Crohn's disease $(\times 300)$.

Fig 5 Crohn's disease. Histologically affected area of the bowel after incubation with anti-IgA. There is an almost complete absence of IgA-containing cells in the lamina propria and little IgA is seen in the epithelium $(\times 90)$

tions, greater than in sections from normal controls. Similarly the low number of IgA-containing cells was not apparently due to the administration of azathioprine, a drug which reduces the immunocyte population of the bowel (Campbell, Skinner, Hersey, Roberts-Thomson, McLennan, and Truelove, 1974), for both the normal population of such cells in the histologically unaffected areas of the bowel and the lack of any correlation between the results noted in individual patients and their antecedent therapy argue strongly against this possibility. It is highly unlikely that the loss of IgA-containing cells was simply a non-specific result of inflammation for this does not occur in cases of acute enteritis (Søltoft and Søeberg, 1972).

The observed abnormality of the secretory immunoglobulin system in Crohn's disease does not therefore appear to be an artefact; it is, however, a curiously focal abnormality, for serum levels of IgA are usually normal in patients with Crohn's disease (Bergman, Johansson, and Krause, 1973) and the histologically unaffected areas of the bowel contain a normal population of IgA-containing cells. This focal loss of IgA could be due to either a failure of

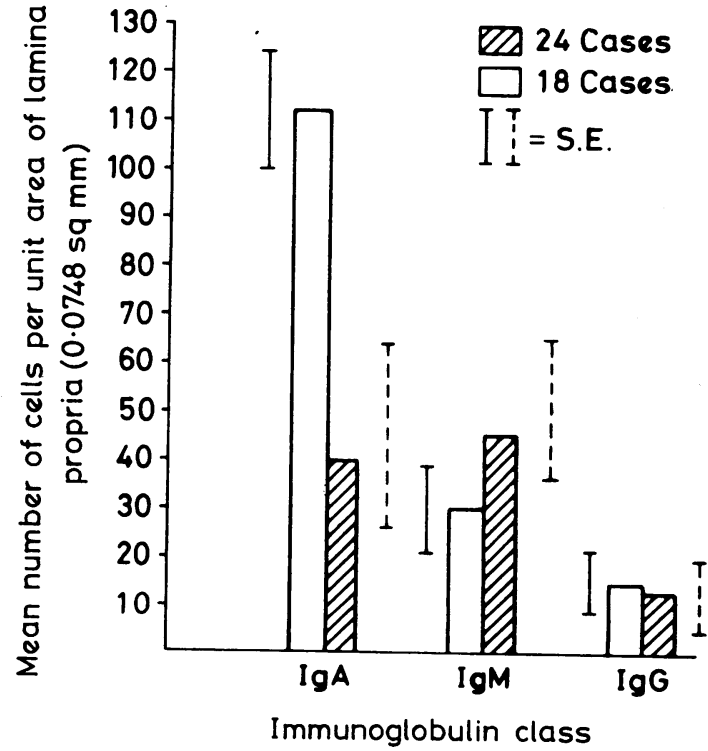

Fig 6 Mean number of immunoglobulin-containing cells in the histologically affected areas of the bowel of patients with Crohn's disease compared with normal controls. 


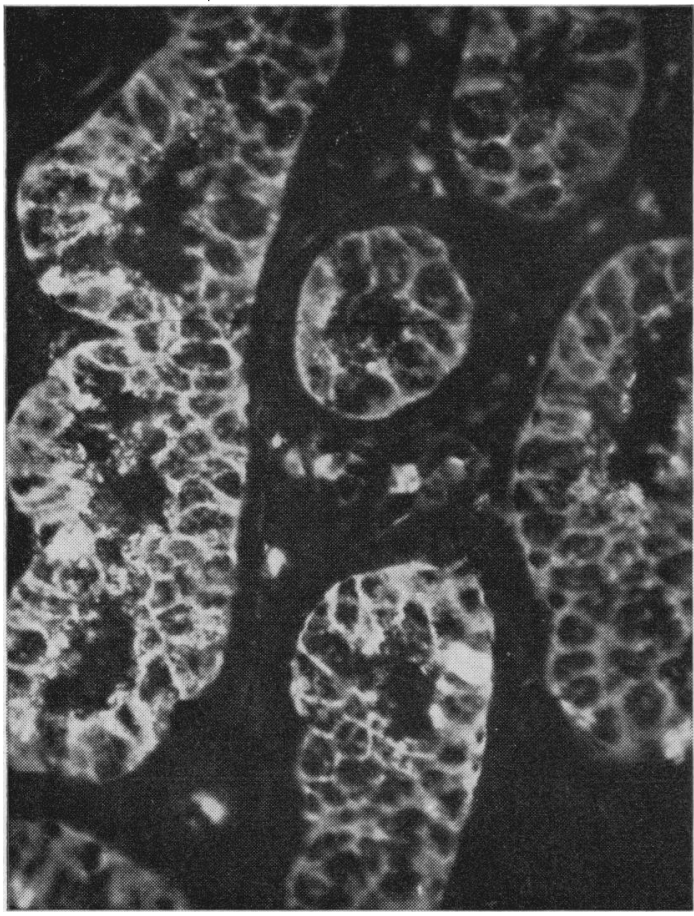

Fig 7 Immunofluorescent localization of secretory piece in the epithelial cells of affected area of the bowel of a patient with Crohn's disease $(\times 300)$.

local synthesis by immunoglobulin-producing cells or to a focal lack of bowel colonization by such cells. There is some evidence that a cell-mediated immune reaction tends to inhibit local humoral immune mechanisms (Feldmann, 1973); there is histological and other evidence to suggest that a cell-mediated immune reaction occurs in the bowel of patients with Crohn's disease (Dykes, 1970; Richens, Williams, Gough, and Ancill, 1974a), albeit possibly a disordered and ineffective one (Aluwihare, 1972; Richens et al, 1974b), and it could therefore be argued that this locally inhibits synthesis of IgA. On the other hand, it is at least equally possible that the reduction in IgA-containing cells is due to a deflection of circulating primed lymphocytes away from the affected areas; the localization of these cells to the gut in germ-free animals is thought to be mediated by a surface receptor recognition system (Griscelli, Vassalli, and McCluskey, 1969; Hall and Smith, 1970), a process termed 'ecotaxis' by de Sousa (1971). This ecotactic system could be altered either by a metaplasia of the surface epithelium, thus altering the specific receptors, or by a masking of the surface receptors

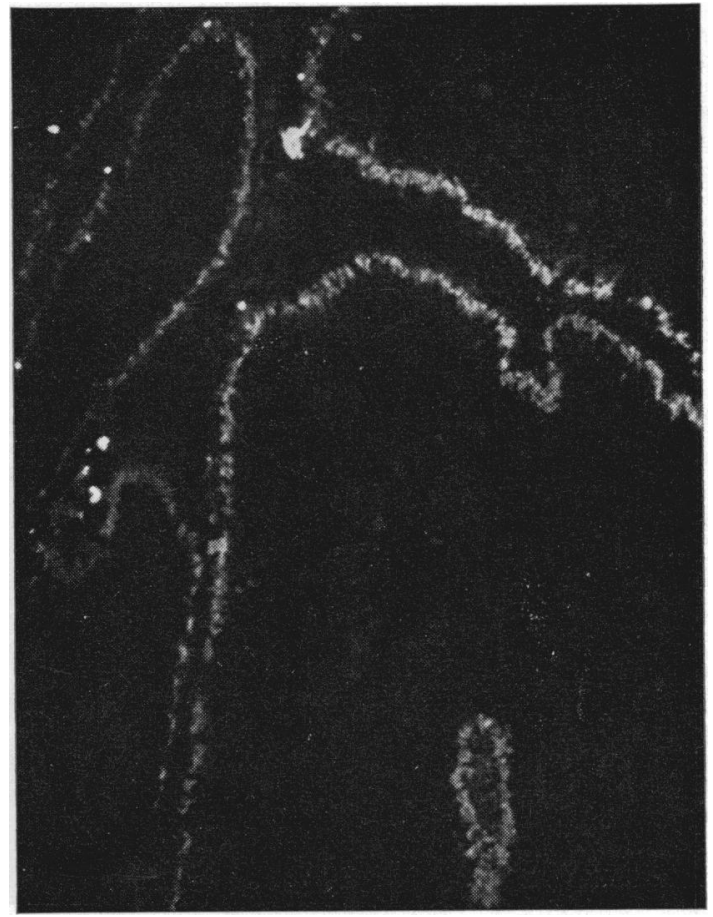

Fig 8 Crohn's disease. Histologically affected area of the bowel after incubation with anti-IgG. There is marked epithelium fuorescence $(\times 90)$.

by bound immunoglobulins. Surface metaplasia is a common phenomenon in Crohn's disease (Whitehead, 1973) whilst our finding that IgG is bound to the epithelial cells of the affected areas of the bowel in many cases may also be of considerable relevance. The IgG appeared to be both on the cell surfaces and within the apical region of the epithelial cells; this immunoglobulin is not, with the possible exception of the female genital tract (Tourville, Ogra, Lippes, and Tomasi, 1970), usually associated with mucosal surfaces but it is possible that the deposition of IgG represented binding to intestinal epithelial antigens of the well documented anticolon antibodies that are found in many patients with Crohn's disease (Dykes, 1972).

Irrespective of the mechanism by which the local decrease in $\operatorname{IgA}$ is achieved, the effect will be a focal deficiency in the 'mucosal block'; there is considerable evidence that locally produced IgA plays a dominant role in the maintenance of this barrier to antigenic material (Shorter, Huizenga, and Spencer, 1972; Walker, Isselbacher, and Bloch, 1972; Williams and Gibbons, 1972) possibly by decreasing antigenic adhesiveness to mucosal cells (Williams and Gibbons, 
1972) or by forming non-absorbable stable complexes with antigens (Heremans and Vaerman, 1971). Crabbe and Heremans (1966) noted a similar local deficiency in some cases of steatorrhoea and considered that this would lead to a focal entry of antigenic material. Whilst the relative lack of IgA would, in itself, increase the permeability of the intestinal epithelium to antigenic material, this effect may be further accentuated by the epithelial binding of IgG which appears both to interfere with $\operatorname{IgA}$ activity, as is the case in herpes simplex keratitis (Centifanto and Kaufman, 1971), and to increase the uptake of antigens (Bockman and Winborn, 1966).

A locally increased intestinal permeability to antigenic material offers an explanation of many of the pathological features of Crohn's disease. Thus the maximal localization of the inflammatory process to the subepithelial area of the gut could be due to the deep penetration of antigenic material whilst the protean nature of the inflammatory response could be due to the gut reacting in diverse fashion to a wide range of antigens; thus, entry of pyogenic organisms could lead to fissuring ulcers and intramural abscesses, penetration of vegetable matter could stimulate the formation of foreign body granulomata, and bacterial capsular antigens could produce tuberculoid granulomata. This concept of altered mucosal permeability is in accord with the recent electron optical demonstration of clusters of different bacteria deep to the intact intestinal mucosa in patients with Crohn's disease (Aluwihare, 1972), a finding which indicates that the entry of antigenic material into the gut wall is not simply a secondary result of ulceration.

\section{References}

Aluwihare, A. P. R. (1972). The electron microscope and Crohn's disease. Clin. Gastroent., 1, 279-294.

Bergman, L., Johansson, S. G. O., and Krause, U. (1973). The immunoglobulin concentrations in serum and bowel secretions in patients with Crohn's disease. Scand. J. Gastroent., 8, 401-406.

Bockman, D. E., and Winborn, W. B. (1966). Light and electron microscopy of intestinal ferritin absorption; observations in sensitized and non-sensitized hamsters (mesocricetus auratus). Anat. Rec., 155, 603-622.

Campbell, A. C., Skinner, J. M., Hersey, P., Roberts-Thomson, P. McLennan, I. C. M., and Truelove, S. C. (1974). Immunosuppression in the treatment of inflammatory bowel disease. I. Changes in lymphoid subpopulations in the blood and rectal mucosa following cessation of treatment with azathioprine. Clin. exp. Immunol., 16, 521-533.

Centifanto, Y. M., and Kaufman, H. E. (1971). The development of tear antibodies and herpes simplex keratitis. In The Secretory
Immunologic System, edited by D. H. Dayton, P. A. Small, Jr., R. M. Chanock, H. E. Kaufman, and T. B. Tomasi, Jr., pp. 331-340. National Institute of Child Health and Human Development. Bethesda, Md.

Crabbe, P. A., and Heremans, J. F. (1966). Lack of gamma A immunoglobulin in serum of patients with steatorrhoea. Gut, 7, 119-127.

de Sousa, M. (1971). Kinetics of the distribution of thymus and marrow cells in the peripheral lymphoid organs of the mouse; ecotaxis. Clin. exp. Immunol., 9, 371-380.

Dykes, P. W. (1970). Delayed hypersensitivity to a faecal antigen in patients with Crohn's disease. (Abstr.) Advance Abstracts of Proceedings of the IVth World Congress of Gastroenterology, Copenhagen, edited by P. Riis, P. Anthonisen, and H. Baden, p. 386. Danish Gastroenterological Association, Copenhagen.

Dykes, P. W. (1972). Immunology of Crohn's disease. Clin. Gastroent., $1,349-366$.

Feldmann, M. (1973). Induction of B cell tolerance by antigen specific $T$ cell factor. Nature [new biol.], 242, 82-84.

Griscelli, C., Vassalli, P., and McCluskey, R. T. (1969). The distribution of large dividing lymph node cells in syngeneic recipient rats after intravenous injection. J. exp. Med., 130, 1427-1451.

Hall, J. G., and Smith, M. E. (1970). Homing of lymph-borne immunoblasts to the gut. Nature (Lond.), 226, 262-263.

Heremans, J. F., and Vaerman, J. P. (1971). Biological significance of IgA antibodies in serum and secretions. Progr. Immunol., 1, 875-890.

Lockhart-Mummery, H. E., and Morson, B. C. (1964). Crohn's disease of the large intestine. Gut, 5, 493-509.

Richens, E. R., Williams, M. J., Gough, K. R., and Ancill, R. J. (1974a). Leucocyte migration studies in Crohn's disease using Crohn's colon homogenate and mitochondrial and microsomal fractions. Gut, 15, 19-23.

Richens, E. R., Williams, M. J., Gough, K. R., and Ancill, R. J. (1974b). Mixed-lymphocyte reaction as a measure of immunological competence of lymphocytes from patients with Crohn's disease. Gut, 15. 24-28.

Schofield, P. F., Deodhar, S. D., and Turnbull, R. B. (1969). Immunoglobulins in non-specific ulcerative disease of the colon; a preliminary report. J. roy. Coll. Surg. Edinb., 14, 157-160.

Schofield, P. F., and Fox, H. (1967). The diagnosis of Crohn's disease of the colon; a clinico-pathological study. Brit. J. Surg., 54, 307-311.

Shorter, R. G., Huizenga, K. A., and Spencer, R. J. (1972). A working hypothesis for the etiology and pathogenesis of nonspecific inflammatory bowel disease. Amer. J. dig. Dis., 17, 1024-1032.

Søltoft, J. (1969). Immunoglobulin-containing cells in normal jejunal mucosa and in ulcerative colitis and regional enteritis. Scand. J. Gastroent., 4, 353-360.

Søltoft, J., and Søeberg, B. (1972). Immunoglobulin-containing cells in the small intestine during acute enteritis. Gut, 13, 535-538.

Tomasi, T. B., Jr. (1970). Structure and function of mucosal antibodies. Ann. Rev. Med., 21, 281-298.

Tomasi, T. B., Jr., and DeCoteau, E. (1970). Mucosal antibodies in respiratory and gastrointestinal disease. Advanc. intern. Med., 16, 401-425.

Tourville, D. R., Ogra, S. S., Lippes, J., and Tomasi, T. B., Jr. (1970) The human female reproductive tract: immunohistological localization of $\gamma \mathrm{A}, \gamma \mathrm{G}, \gamma \mathrm{M}$, secretory 'piece' and lactoferrin. Amer. J. Obstet. Gynec., 108, 1102-1108.

Walker, W. A., Isselbacher, K. J., and Bloch, K. J., (1972). Intestinal uptake of macromolecules: effect of oral immunization. Science, 177, 608-610.

Whitehead, R. (1973). Mucosal Biopsy of the Gastrointestinal Tract. (Major Problems in Pathology, Vol. 3, p. 123. Saunders, London, Philadelphia.

Williams, R. C., and Gibbons, R. J. (1972). Inhibition of bacterial adherence by secretory immunoglobulin A: a mechanism of antigen disposal. Science, 177, 697-699.

Williams, W. J. (1964). Histology of Crohn's syndrome. Gut, 5, 510-516. 